\section{Infección respiratoria causada por Alcaligenes xylosoxidans en un paciente con síndrome de Mounier-Kuhn}

\author{
Marta Arroyo-Cózar, Montserrat Ruiz-García, \\ Eva M. Merlos, David Vielba y Enrique Macías
}

Case report: respiratory infection due to Alcaligenes xylosoxidans in a patient with Mounier-Kuhn syndrome

Mounier-Kuhn syndrome is a rare entity characterized by abnormal dilatation of the trachea and main bronchi (tracheobronchomegaly). Alcaligenes xylosoxidans is a non fermenting gram-negative pathogen common in extra-and intra-hospital environment, which may be related to immunosuppression states. We describe the case of a 75 years old male, ex-smoker with moderate functional obstruction, chronic respiratory failure and chronic colonization by Pseudomonas aeuriginosa. He had an infectious exacerbation of his disease, reason that previously required several hospital admissions. The patient was treated with antibiotics and his evolution was favourable with negativization in cultures of the pathogen. This is the first description of the isolation of Alcaligenes xylosoxidans as a cause of respiratory infection in a patient with Mounier-Kuhn syndrome.

Key words: Mounier-Kuhn syndrome, tracheobronchomegaly, Alcaligenes xylosoxidans, respiratory infection.

Palabras clave: Síndrome de Mounier-Kuhn, traqueobroncomegalia, Alcaligenes xylosoxidans, infección respiratoria.

\section{Introducción}

$\mathrm{E}$ 1 síndrome de Mounier-Kuhn está caracterizado por la dilatación anormal de la tráquea y los bronquios principales (traqueobroncomegalia). Se asocia a enfermedad bronquiectásica difusa congénita de predominio en lóbulos inferiores, además de a infecciones de repetición del tracto respiratorio inferior ${ }^{1}$. Es una entidad rara, de la que no se conoce su prevalencia con exactitud, ya que existen muchos pacientes asintomáticos o con sintomatología leve, por lo que se sospecha que su incidencia pudiera ser mayor que el centenar de casos descritos ${ }^{2,3}$. Se diagnostica más frecuentemente en varones entre los 30-40 años y es habitual la predisposición a presentar otras patologías pulmonares asociadas ${ }^{4}$.

Describimos el primer caso de infección pulmonar por Alcaligenes xylosoxidans en un paciente con síndrome de Mounier-Kuhn.

\footnotetext{
Hospital General Universitario de Elche, Elche, Alicante, España.

Servicio de Neumología (MAC).

Servicio de Microbiología (MRG).

Servicio de Medicina Familiar y Comunitaria (EMM).

Hospital Clínico Universitario de Valladolid, Valladolid, España.

Servicio de Neumología (DV, EM)

Recibido: 29 de noviembre de 2011 / Aceptado: 14 de mayo de 2011
}

Correspondencia a:

Marta Arroyo-Cózar

m arroyo cozar@hotmail.com

\section{Caso clínico}

Varón de 75 años, ex-fumador de 70 paquetes al año de consumo acumulado, con síndrome de Mounier-Kuhn diagnosticado hacía aproximadamente 30 años, con obstrucción funcional moderada e insuficiencia respiratoria crónica tipo 1 con oxígenoterapia domiciliaria continua. Tenía antecedentes de colonización pulmonar crónica por Pseudomonas aeruginosa desde hacía dos años, en tratamiento con colistimetato de sodio inhalado, y varios hospitalizaciones por exacerbación de su patología de base.

Durante una internación por síncope presentó aumento de la tos y expectoración habituales con cambio en la coloración y consistencia (verdosa purulenta) de la secreción bronquial, aumento de disnea hasta hacerse de reposo y fiebre de $38^{\circ} \mathrm{C}$. En la exploración física, se encontraba eupneico, sin tiraje, frecuencia respiratoria de $14 \mathrm{rpm}$ y saturación de oxígeno de $98 \%$ con cánula nasal a $2 \mathrm{~L} / \mathrm{min}$. En la auscultación pulmonar destacaban roncus y crepitaciones posteriores en los dos tercios inferiores bilaterales. Exámenes de laboratorio: hemograma con 12.940 leucocitos $/ \mathrm{mm}^{3}$ con neutrofilia de $79 \%$. Gasometría arterial con oxígenoterapia a $2 \mathrm{~L} / \mathrm{min}$ : $\mathrm{pH} 7,4 ; \mathrm{pCO}_{2} 34$ $\mathrm{mmHg}, \mathrm{pO}_{2} 59 \mathrm{mmHg}$, saturación de oxígeno $91 \%$ y bicarbonato $22 \mathrm{~mm} / 1$. La radiografía torácica mostraba un patrón intersticial reticular bilateral en los lóbulos inferiores, probables bronquiectasias y ensanchamiento mediastínico por aumento del calibre de la tráquea y bronquios principales. En la TAC torácica había signos de enfisema centrolobulillar y paraseptal en los lóbulos superiores con grandes bullas confluentes, dilatación de tráquea y bronquios principales y numerosas bronquiectasias con predominio en lóbulos infe-riores, asociando varios focos parcheados de aumento de densidad (Figuras 1 a 4). Se obtuvieron dos muestras de expectoración, en días sucesivos, al inicio del cuadro clínico, con una aceptable calidad de la muestra medida por la presencia de moderados leucocitos en la tinción de Gram. Fueron sembradas en medios de cultivo habituales, obteniéndose crecimiento abundante de colonias de $A$. xylosoxidans. La identificación se realizó mediante paneles semiautomatizados del sistema Wider y se confirmó en el laboratorio de referencia correspondiente. En el estudio de susceptibilidad, resultó sensible a carbapenem, piperacilina/tazobactam, cotrimoxazol y gentamicina; resistente a cefalosporinas, tobramicina, aztreonam y ciprofloxacina. Se inició terapia con piperacilina-tazobactam iv ( 4 g cada 8 h) y azitromicina empíricamente, además de broncodilatadores inhalatorios, corticoesteroides sistémicos y oxígenoterapia. Evolucionó favorablemente, afebril y con disminución progresiva de la disnea y la tos. La expectoración se volvió más clara y fluida y recuperó cifras de saturación normales con su nivel de oxígenoterapia habitual a los 10 días de tratamiento antibacteriano iv.

Se dio de alta con tratamiento oral con cotrimoxazol (800/160 mg cada 12 h) durante 12 días más. En las muestras posteriores no se aisló A. xylosoxidans y sólo había microbiota habitual del tracto respiratorio.

\section{Discusión}

Alcaligenes xylosoxidans, también conocido como Achromobacter xylososidans, es un bacilo gramnegativo patógeno no fermentador ${ }^{5}$, frecuente en el ambiente extra e intrahospitalario. Está relacionado con estados de inmunosupresión ${ }^{6}$, como en este caso, por ciclos prolongados de antibioterapia y corticoesteroides. Coloniza el tracto respiratorio de niños intubados y de pacientes con fibrosis quística ${ }^{7}$, pero excepcionalmente se han descrito infecciones del aparato respiratorio. Presenta resistencia frecuente a aminoglucósidos y quinolonas ${ }^{8}$. Sin embargo, la sensibilidad presenta un umbral bajo a carbapenem, $\beta$-lactámicos con acción antipseudomonas y cotrimoxazol, en casi el total de series publicadas?. 


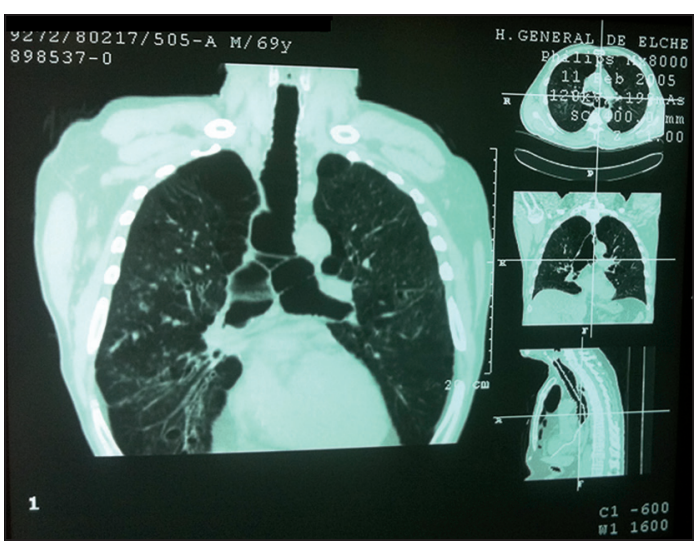

Figura 1. TAC pulmonar con reconstrucción; vista anteroposterior: dilatación de traquea y bronquios principales.

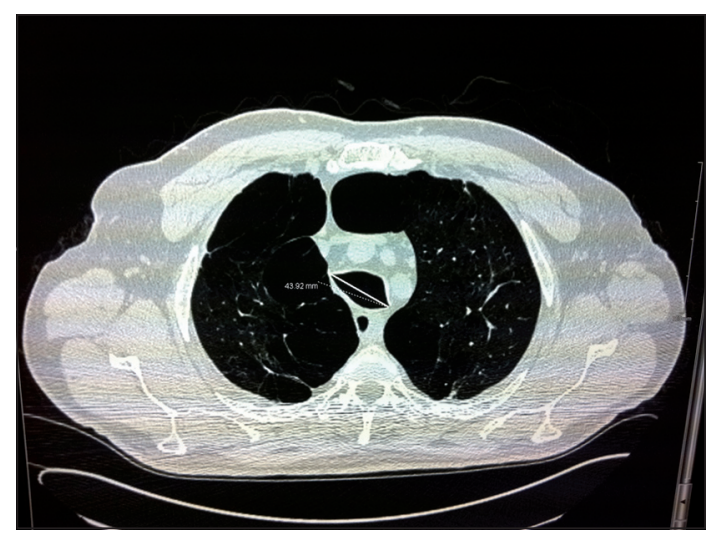

Figura 3. TAC pulmonar: dimensiones de la traquea.

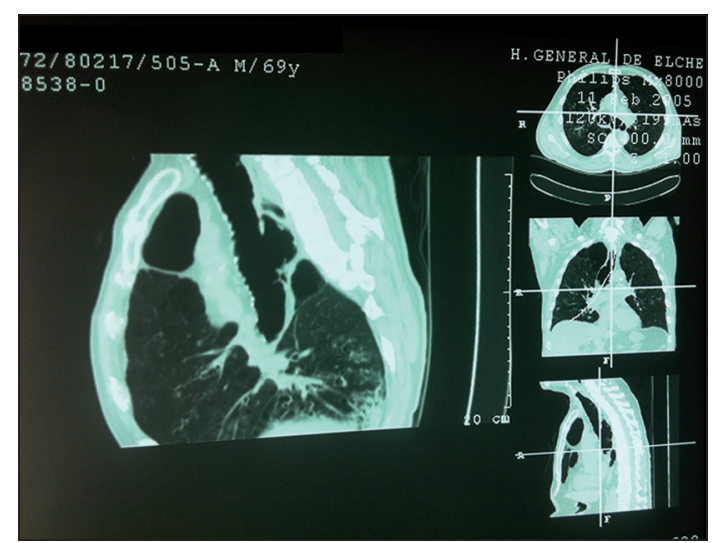

Figura 2. TAC pulmonar con reconstrucción; vista lateral: dilatación de traquea y bronquios principales.

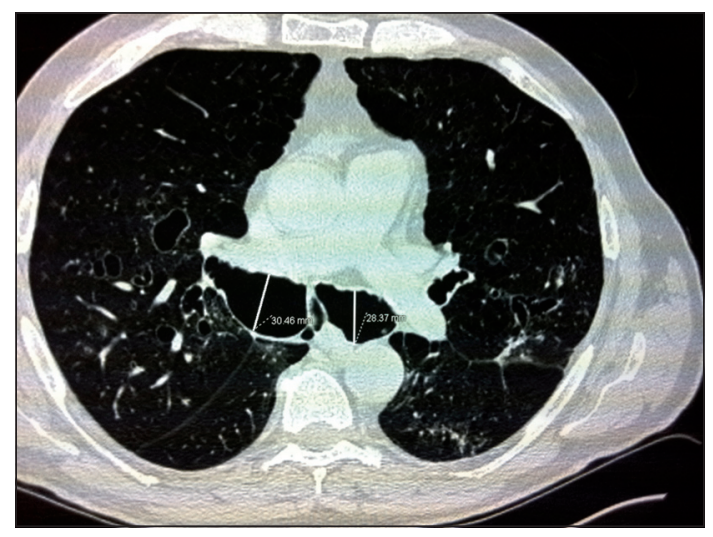

Figura 4. TAC pulmonar: dimensiones de los bronquios principales.

\section{Referencias bibliográficas}

1. Celik B, Bilgin S, Yuksel C. Mounier-Kuhn syndrome: a rare cause of bronchial dilation. Tex Heart Inst J 2011; 38: 194-6.

2. Schwartz M Rossoff Tracheobronchomegaly. Chest 1994; 106 : 1589-90.

3. Min J. Lee JM, Kim JH, Hong DM, Jeon Y, Bahk JH Anesthetic management of a patient with Mounier-Kuhn syndrome undergoing off-pump coronary artery bypass graft surgery- A case report. Korean J Anesthesiol 2011; 61: 83-7.

4. Jareño J J, de Granda J I, Campos S, Villegas F, Zuil M, Gutiérrez T, Traqueobroncomegalia y bronquiectasias en el síndrome de Mounier-Kuhn. Colonización por Pseudomonas aeruginosa y antibioterapia inhalada. Rev Patol Resp 2010; 13: 127-9.

5. Lambiase A Catania MR Del Pezzo M Rossano H Terlizzi et al. Achromobacter xylosoxidans respiratory tract infection in cystic fibrosis patients. Eur J Clin Microbiol Infect Dis 2011; 30: 973-80.

6. Duggan JM Goldstein S. Chenoweth CE, Kauffman CA, Bradley SA Achromobacter xylosoxidans bacteremia: report of four cases and review of the literature. Clin Infect Dis 1996; 23: 569-76.

7. Dunne WM Ju Maisch S. Epidemiological investigation of infections due to Alcaligenes species in children and patients with cystic fibrosis: use of repetitive-element-sequence polymerase chain reaction. Clin Infect Dis 1995; 20: 836-41.

8. Magni A Giordano A Mancini 0 , Pecoraro O, Varesi 1 , Quattrucci S, et al. Emerging cystic fibrosis pathogens: incidence and antimicrobial resistance. New Microbiol 2007; 30: 59-62.

9. Sancho-Chust JN Agudo A, Camarasa A, Chiner B. Achromobacter xylosoxidans como agente colonizador en bronquiectasias. Enferm Infecc Microbiol Clin 2010; 28: 203-4. 